\title{
L'enfant chargé de songes et de lectures
}

AnNie Tanguay

UNIVERSITÉ DE SHERBROOKE

plus ou moins, tous les livres, contiennent la fusion de quelques redites comptées ${ }^{1}$

Stéphane Mallarmé

Résumé. Cet article examine plusieurs références et allusions intertextuelles et intratextuelles dans L'enfant chargé de songes d'Anne Hébert. En plus d'en dégager la diversité et la richesse, il montre en quoi ces références et allusions éclairent le fonctionnement du roman. La plupart des intertextes sont issus des lectures d'enfance et de jeunesse de l'auteure. Plusieurs proviennent de textes poétiques d'auteurs qui l'ont marquée durablement : Rimbaud, Claudel et Baudelaire.

Mots-clés : L'enfant chargé de songes, Roman poétique, Intertextualité, Intratextualité, Lectures et influences.

En 1992, Anne Hébert publie son septième roman, L'enfant chargé de songes, que I'on peut qualifier à plusieurs égards de roman poétique. Il répond aux définitions ${ }^{2}$ qu'en donnent Jean-Yves Tadié - quant à la structure circulaire -, et de Karlheinz Stierle - quant à la prédominance du discours sur I'histoire. Plusieurs autres composantes peuvent être relevées ${ }^{3}$, dont une forte présence de l'intertextualité, qui fait entrer la voix d'Anne Hébert en dialogue avec celles d'autres créateurs. C'est ce rapport spécifique à l'intertextualité, au sens que donne à ce mot Gérard Genette, en tant que « relation de coprésence entre deux ou plusieurs textes, c'est-à-dire, [...] la présence effective d'un texte dans un autre » (Genette, $1982: 8$ ), que je développerai dans le présent article. Un tel métissage prend différentes formes : la citation, le plagiat (qui relève d'emprunts où le nom de l'auteur n'est pas mentionné) et l'al-

1. Voir « Crise de vers », texte paru en 1897, repris dans les Divagations (Mallarmé, $1993: 250$ ).

2. Voir Tadié, $1994: 7$ et suivantes, et Stierle, $1977: 431$.

3. L'illusion référentielle est constamment brisée par l'aspect dominant du rêve et du fantasme. Le texte est fragmenté. Les retours dans le temps y sont fréquents. 
lusion. On relèvera ainsi bon nombre d'intertextes issus des lectures d'enfance et de jeunesse, poèmes ou romans, ainsi que plusieurs références à la Bible, notamment au récit de la Création, qui confèrent un aspect mythique aux personnages.

Si les références à la Bible et aux écrivains sont abondantes, il ne faut pas négliger pour autant les marques, tout aussi foisonnantes, d'intratextualité ${ }^{4}$ dans L'enfant chargé de songes. L'abondance de ces marques permet de mesurer à quel point le roman s'inscrit dans la continuité de l'œuvre et se présente comme une synthèse des lectures marquantes d'Anne Hébert.

Qu'elles soient intratextuelles ou intertextuelles, toutes ces marques convergent pour dire une certaine nostalgie de l'enfance et du premier séjour en France de l'auteure en $1954^{5}$. On peut affirmer en ce sens que L'enfant chargé de songes est le résultat d'un travail sur les traces que laissent les écrits. On trouve dans ce roman plusieurs références à I'œuvre poétique de Rimbaud et à la Bible, qu'elle apporterait avec elle sur une île déserte (Fortin, 1992 : 22). Sont également présents dans le texte, par la mention de leur nom ou par la citation d'extraits de leurs œuvres, les Baudelaire, Mauriac, Dickens, Supervielle et Claudel, auteurs qu'elle a découverts par I'entremise de son cousin Saint-Denys Garneau vers I'âge de 18 ou 20 ans (Montalbetti, 1969 : 39; Salgas, 1985 : 18). On y retrouve également certains contes de son enfance, dont ceux de Perrault et d'Andersen, qui ont, au dire de l'auteure, contribué à la création de son imaginaire (Royer, 1980 : 21). Les romanciers Colette et Faulkner sont à leur tour convoqués. Dans ce roman, l'intertextualité est, plus que jamais dans l'œuvre, diversifiée. Cela tient sans doute au fait que le personnage principal est, comme l'auteure, un grand lecteur. Devant un tel foisonnement de références littéraires, il importe de faire un relevé exhaustif, pour en dégager une sorte de poétique et I'hommage rendu à ceux et celles qui I'ont inspirée. Mais avant d'entrer dans le texte à proprement parler, je m'emploierai à expliquer le titre de ce roman, révélateur d'un travail aussi bien intertextuel qu'intratextuel.

4. L'intratextualité correspond à des références qu'un auteur fait à ses propres textes.

5. Anne Hébert enrichira la vie de Julien de ses expériences relatives à son premier séjour à Paris, qu'elle détaille dans sa prose «Mon cœur sauvage, je le dis en français » parue dans Les nouvelles littéraires en janvier 1976, ainsi que de celles de son cousin, qui s'y est rendu en 1937, et dont Marie-Andrée Lamontagne témoigne dans la revue Liberté, en 1993. 


\section{Des ténèbres aux songes}

Dans un carnet d'écriture, Anne Hébert avait envisagé d'autres titres pour ce texte : «Une leçon de ténèbres », « La géante » et «Ardentes ténèbres » (Watteyne, 2008 : 71), qui rappellent des poèmes du recueil Le jour n'a d'égal que la nuit, publié la même année que le roman. « Leçon de ténèbres » figure dans la section « Poèmes nouveaux 1987-1989 ». Le poème, tout comme les compositions du même nom de François Couperin inspirées du texte biblique « Les lamentations de Jérémie », renvoie à l'une des scènes de la première partie du roman, où Julien assiste à un concert : « Julien est livré à la musique, sa propre nuit débusquée ne fait plus qu'une nuit rayonnante avec la Leçon de ténèbres, célébrée dans la petite église des Billettes. Rien au monde, semble-t-il, ne peut distraire Julien de son recueillement. » (L'enfant chargé de songes, 1999 : 15) Julien est à l'image du sujet du poème, qui « S'endor[t] debout / Comme un arbre / Dans la nuit ». (Fuvres complètes I, 2013 : 374) Mais, durant son recueillement, il est déconcentré par la présence de Camille, qui lui rappelle Lydie. Camille n'est-elle pas cette petite étoile ${ }^{6}$ qui, dans le poème, redonne un peu d'espoir au sujet lyrique? Par la médiation de ce personnage féminin, Julien replongera dans son passé et il pourra enfin s'en distancier après avoir assouvi un désir longtemps réprimé. En couchant avec Camille, il pourra en effet se dégager de l'emprise de Lydie. Il ne se libérera pas tout à fait de son passé, mais Iorsqu'il retournera au Québec, ce sera pour lui un nouveau départ. Du moins est-ce ainsi que nous le présente l'auteure dans les dernières lignes de son roman.

Il est possible de comprendre l'hésitation de l'auteure pour un titre comme « La géante », lorsqu'on lit « Apparition », le dernier texte de la section « Poèmes anciens 1961-1980». Le contenu de ce poème se rapproche en effet de l'ouverture du roman de 1992. Julien est livré alors « aux apparitions de la nuit » : « Soudain elle a été là, dans les ténèbres de la chambre, de plus en plus nette et précise, à mesure qu'il la reconnaissait. Bientôt la géante immobile et lourde s'est mise à rayonner de mauvaise humeur et Julien a su que sa mère ne lui pardonnait pas d'avoir franchi I'Atlantique et quitté sa terre natale. » $\left(E C S^{7}: 9\right)$ L'aspect mortifère de sa relation avec Pauline ressort ainsi dès les premières phrases du roman. Dans le poème, une dynamique similaire est reprise : « Ayant atteint sa taille parfaite / La géante bouge

6. Étoile évoquée dans la dernière strophe du poème : «Immobile / Attendre que les temps soient révolus / Dans l'espoir d'une petite étoile / À I'horizon couleur de suie. 》 (Fuvres complètes I, $2013: 374)$

7. ECS pour L'enfant chargé de songes, 1999. 
dans l'ombre / Et fait vaciller la chambre sous son poids ». (Poésie : 356) Julien est présenté comme prisonnier de ses ténèbres intérieures. Dans d'autres œuvres d'Anne Hébert, les mères sont perçues par leur fils comme des géantes. Dès les premières lignes de la nouvelle «Le torrent », Claudine bénéficie d'un tel éclairage : « Je voyais la grande main de ma mère quand elle se levait sur moi, mais je n'apercevais pas ma mère en entier, de pied en cap. J'avais seulement le sentiment de sa terrible grandeur qui me glaçait. » (Le torrent, 1972 : 9-10)

Anne Hébert retient finalement un titre qui présente un lien explicite avec L'enfant chargé de chaînes (1913) de François Mauriac, auteur qu'Hébert lisait dans son adolescence. Certains éléments du récit configuré par elle se rapprochent de ceux élaborés par son prédécesseur, soixante-dix ans plus tôt, à savoir que l'enfance est un lieu difficile, voire impossible à quitter. Dans le roman de Mauriac, Jean-Paul, le héros, est, tout comme Julien, un jeune homme solitaire qui a une passion pour la littérature. Il éprouve lui aussi de la difficulté à rompre avec le passé et parle de son « passé, dont [il est] obsédé » (Mauriac, 1978 : 33), de ses souvenirs « redoutés et adorés », qu'il souhaite fuir, puisqu'« ils le surprennent à chaque heure de la journée. » (Mauriac, 1978 : 52) À la fin du roman, Jean-Paul s'adresse à Marthe, la femme dont il se dit amoureux : «Vous savez que le bien-aimé demeure malgré tout un enfant chargé des chaînes et qu'il n'est pas encore délivré... » (Mauriac, 1978 : 79) Chez Hébert, les songes de Julien le retiennent dans son enfance au même titre que des chaînes. Par la métaphore des songes, l'auteure innove en faisant état des fantasmes de Julien, tandis que Mauriac ne creuse que très peu l'intériorité de son personnage. Les chaînes ne laissent aucune ambigüité sur l'état de prisonnier de Jean-Paul, mais le malaise de Julien n'est pas suggéré de la même façon. Par la similarité des deux titres, un lecteur averti ne peut qu'y voir l'impossibilité d'une libération totale pour le protagoniste hébertien.

Au tout début de L'enfant chargé de songes, un autre rapprochement est fait entre Julien et le Faust ${ }^{8}$ (1808) de Gœthe : " "Salut, mon premier matin à Paris!", chantonne Julien, $[. .$.$] devant sa glace, retrouvant un air de Faust, tout en essayant de$ mettre de l'ordre dans sa chevelure très frisée où s'emmêlent quelques fils d'argent. » (ECS : 11) Le Faust de Goethe est un personnage épris de connaissances. Pris en charge par Méphistophélès, I'un des princes de l'enfer, il sera initié aux jouissances terrestres à la condition de livrer son âme au diable. Et c'est bien ce que fait Julien,

8. L'aspect musical peut renvoyer aussi à I'opéra de Charles Gounod (1859), inspiré du célèbre roman de Gœthe. 
quant à lui livré à son « mauvais génie » (ECS : 59), Lydie, présentée comme son premier et seul amour. Elle lui avait apporté le goût de la littérature et de la musique. Il en reste prisonnier bien des années plus tard. Julien revit toujours en songes les quelques semaines qu'ils ont passées ensemble, mais n'a pas oublié le tort qu'elle a causé à sa famille.

Souvenirs, souvenirs...

Anne Hébert dit avoir lu dans son enfance beaucoup de contes de fées et I'histoire sainte (Smith, 1983 : 42). Initiée très jeune à la littérature, d'abord par l'entremise de ses parents, qui lui faisaient la lecture, elle développe sa capacité à rêver, condition essentielle, selon elle, pour devenir un écrivain (Royer, 1980 : 21). On rencontre dans tous ses textes des enfants et des adolescents. À Jean-Paul Kauffmann, qui lui demandait si elle connaissait déjà le sujet de son prochain roman après Kamouraska, elle répond : « Je n'ai pas trouvé encore de sujet. Il y sera certainement question de l'enfance ou de l'adolescence, ce sont des choses qui vous marquent pour toute la vie. » (Kauffmann, $1970: 10$ ) Aussi estime-t-elle que « les souvenirs d'enfance d'un écrivain sont souvent à l'origine des fantasmes qui hantent son œuvre. » (Escomel, 1980 : 10) En écrivant L'enfant chargé de songes, Anne Hébert fait d'ailleurs un retour sur sa propre enfance, évoquant les étés passés à la campagne, les vastes étendues, les couleurs et les odeurs qui s'y déploient. Elle revisite également différents textes lus dans sa jeunesse.

Don Quichotte, que son père lui lisait, est un fou de littérature, dévoreur de romans chevaleresques, qui part à la découverte du monde afin de voir si la réalité corrobore ses lectures. À ce point convaincu de la vérité des textes, ne pouvant admettre qu'il s'illusionne, il s'imagine des opposants et des assaillants à repousser. Lorsque Julien est comparé à « un Don Quichotte enfantin, gorgé de lectures et de musiques, qui accumule des trésors, avant de partir pour le vaste monde » (ECS : 136), cela n'augure rien de bon sur la capacité du personnage à percevoir la réalité. Après la mort de sa mère, il choisit de se réfugier dans les livres, qui le «transport[ent] dans un univers sans limites, foisonnant de sensations étranges et de personnages étonnants » (ECS : 131) qui le protègent, croit-il, des fantômes de son passé. Il s'est créé, par la littérature, un refuge pour contrer sa solitude : «Des villes fabuleuses apparaissent entre les lignes de ses livres, laissent entrevoir le dédale des rues et des ruelles étrangères, tandis que de grandes places sacrées surgissent avec des cathédrales debout, comme des pierres énormes dressées, pleines de saints et de démons sculp- 
tés. » (ECS : 131) Julien souhaite voyager, aller à la rencontre d'autrui, mais le réel n'est pas à la hauteur de ses attentes. À la différence de Don Quichotte, celui-ci se montre vite déstabilisé par le monde qu'il découvre. Il a l'impression que « [I]a vraie ville [...] [est] hors d'atteinte » (ECS : 12), ne correspondant pas à celle entrevue dans les livres. La réalité lui apparaît étouffante, et l'animation de la ville le dérange. Mais qu'est-ce qu'un Don Quichotte comme lui souhaite véritablement découvrir? La littérature et la musique lui avaient autrefois permis d'entrer en communion avec Lydie, comme en témoigne un passage de sa lettre à la jeune femme : « La poésie et la musique illuminent ma vie, l'agrandissent sans fin, me rapprochent de toi à la vitesse de la lumière, me permettent de me retrouver avec toi dans le même éblouissement. » (ECS : 87) À Paris, il espère enfin pouvoir renouer avec les moments les plus agréables de son passé et ressentir à nouveau un semblant de paix. Mais on peut dire qu'il échoue, dans une certaine mesure, car même à la fin du récit, Julien continue de nourrir ses fantasmes, incapable de se satisfaire de la réalité, toujours tourné vers le songe.

Anne Hébert fait état d'autres lectures, autrement plus déstabilisantes, par l'entremise du personnage de Camille, soit la nouvelle de Stevenson « Doctor Jekyll and Mister Hyde » (ECS : 149), ce qui met en relief les différents doubles mis en forme dans le roman. Julien jouit de cette duplicité qui lui permet de se fondre dans la foule : «Une seconde existence double sa petite vie d'employé modèle et d'amant très sage. » (ECS : 131) Il est, face au monde, un homme sérieux et modéré et, dans la solitude, un poète-rêveur. L'existence de Julien vacille ainsi entre la réalité et le songe, entre le monde des vivants et celui des morts. Les femmes qu'il rencontre sont à l'image de celles qu'il a connues. Camille Jouve semble être, à différents égards, la réincarnation de Lydie Bruneau. Quant à Aline, elle revêt la figure de la bonne mère, telle la Pauline de son enfance.

Il est intéressant de remarquer que, dans la plupart des contes de Perrault et d'Andersen, que l'auteure lisait au collège, la femme est présentée comme un être faible qui a besoin d'être secouru, au contraire de plusieurs héroïnes hébertiennes ${ }^{9}$. Le refus d'Aline d'aller vivre chez Julien en l'absence de celui-ci n'est-il pas un signe d'émancipation? Deux chambres lui sont interdites, et elle a l'impression d'entendre chuchoter les mortes qui les habitent. Elle refuse alors de faire partie du cruel conte de Perrault pour jeunes filles, Barbe-Bleue (ECS : 139). Elle veut vivre selon ses

9. L'auteure estime que « cela doit correspondre à [s]a façon de voir la femme qu['elle] ne perçoi[t] pas du tout comme un être fragile, mais comme un être fort. » (Escomel, $1980: 10$ ) 
règles, bien qu'elle tremble à l'idée d'être séparée de son amoureux. Reste qu'Aline n'est pas la femme la plus déterminée de l'œuvre hébertienne... On trouve aussi quelques allusions à Andersen, plus précisément au conte «Les souliers rouges », qu'il a écrit en 1845. Ces souliers rouges ont le pouvoir magique de faire danser sans fin celui qui les porte. À plus d'une reprise, Julien « s'adapte et prend le rythme » (ECS : 13) pour marcher dans Paris en songeant aux chaussures rouges d'Aline. Les chaussures viennent constamment marquer le mouvement : «Ses souliers rouges trottinent allégrement sur le trottoir. » (ECS : 122) La jeune femme s'impose à la mémoire de Julien, en surgissant « au détour d'une rue [...] avec des souliers rouges et une robe à fleurs ». (ECS : 145) C'est par la médiation de ces chaussures qu'il pense à elle, et retrouve son énergie, ce qui, somme toute, lui est bénéfique. Mais I'histoire d'Andersen est beaucoup plus sordide. La seule manière de mettre un terme à l'enchantement est de se mutiler ${ }^{10}$.

Vers l'âge de 20 ans, Anne Hébert a découvert également l'œuvre de Supervielle. Elle retiendra notamment de ce dernier le caractère onirique de certains textes. Dans Les chambres de bois, elle citait, en exergue de la troisième partie, une phrase de Supervielle : « ...une toute petite bague pour le songe » (Les chambres de bois, 1958 : 143) et reprenait la formule au moment de clore le roman : " "Une toute petite bague pour le songe", Michel, rien qu'une toute petite bague. » (CB $\left.{ }^{11}: 190\right)$ En transformant cette citation de Supervielle dans L'enfant chargé de songes, I'auteure crée une relation intratextuelle entre ses deux romans. La citation est alors quelque peu modifiée - «Une petite bague pour le concert, rien que pour le concert » (ECS : 148) -, afin de marquer l'alliance de Camille avec la musique et, de fait, insister sur le caractère sacré de la musique, valorisée dans tout le livre.

Anne Hébert confie avoir lu, dès le collège, tout ce que Colette a écrit, et dit l'avoir relue par la suite. Elle se dit admirative de l'écriture de la romancière française, « de sa façon de faire vivre la nature, bien entendu, mais les bêtes, les femmes, surtout les femmes, parce que les hommes chez Colette sont un peu effacés. » (Jacques

10. Anne Hébert en creusera l'aspect mortifère en 1997 dans le texte « La danseuse tuée » de ses Poèmes pour la main gauche. Comme le fait ressortir Nathalie Watteyne en 2013, en dépit de la mort, « [I]es pas volatiles [de la danseuse tuée] miment sans fin / Des pointes et des entrechats / Sans qu'on puisse les arrêter. » (Fuvres complètes $I$ : 399) Le terrible enchantement ne semble pas avoir de fin. Par ailleurs, dans le poème «Acte de foi », le sujet féminin « s'imagine tenir dans sa main droite / La terre ronde rude obscure / Comme une orange sanguine qui luit » (Fuvres complètes I : 409 ) tout comme Aline qui constate que « le monde entier est là devant elle, rond comme une orange qu'elle pourrait tenir dans sa main. » (ECS : 126)

11. CB pour Les chambres de bois, 1958. 
et Hulot, 1995) Lorsqu'Anne Hébert obtient sa bourse, pour poursuivre des études en France en 1954, Colette vient de mourir. La mère d'Hébert lui aurait dit : «Quel dommage, tu ne connaîtras pas Colette. » (Jacques et Hulot, 1995) Il n'est donc pas anodin de retrouver une référence à cette écrivaine dans un roman empreint de nostalgie qui rappelle, à différents niveaux, son premier voyage en France. Dans le livre de souvenirs L'étoile Vesper, en 1946, Colette se montrait ébahie de la capacité qu'avait George Sand de combiner sa vie personnelle à un œuvre volumineux, capacité qu'elle-même aurait voulu avoir : « Comment diable s'arrangeait George Sand? Cette robuste ouvrière de lettres trouvait moyen de finir un roman, d'en commencer un autre dans la même heure. Elle n'en perdait ni un amant, ni une bouffée de narghilé [...]. Puissamment, elle agença pêle-mêle son travail, ses chagrins guérissables et ses félicités limitées. Je n'aurais pas su en faire autant. » (Colette, 1989 : 682; je souligne) Comment ne pas faire le rapprochement avec la scène d'ouverture de la quatrième partie de L'enfant chargé de songes où Julien envie l'insouciance de Camille Jouve : «Il voudrait n'avoir ni passé ni tourment, que des félicités mesurables et des chagrins guérissables, comme cette femme tout contre lui qui n'en finit pas de rire. » (ECS : 143) Si le passé de Julien cessait de le tourmenter, peut-être pourrait-il avoir une vie agréable, faite d'insouciance, où le plaisir se mêle à la passion. Telle George Sand, Camille semble avoir cette même insouciance que Julien lui reprochera bientôt.

Le bruit et la fureur de William Faulkner est un autre roman qui a marqué Anne Hébert ${ }^{12}$. Juste après l'incident à la rivière où Lydie s'est pratiquement noyée, il est dit de sa tête qu'elle « est pleine de bruit et de fureur » (ECS : 106). L'auteure utilise cette référence pour montrer la puissance de la nature par rapport à la fragilité du corps humain. Les éléments naturels, ici le ressac de l'eau, ne cessent de rappeler leur supériorité. Lydie n'a pu sauver Hélène qui, elle, est emportée par le « roi de la vase ». Diverses allusions, références et variations à partir de ce titre de Faulkner reviennent dans plus d'un texte d'Hébert. L'immensité du paysage et la force qui le caractérise sont mises de l'avant et menacent les personnages qui parfois s'approprient cette fureur, parfois la subissent. Malgré sa force de caractère, Lydie ne réussit pas à dominer la nature.

12. Elle possède trois éditions de ce roman dans sa bibliothèque personnelle, la première étant datée de 1959. 
Outre les lectures de jeunesse, la Bible reste une des principales sources d'inspiration, « peut-être l'œuvre qui [I]'a marquée le plus... » (Vanasse, 1982 : 444) La parole liturgique est à ses yeux « une poésie extraordinaire » (Dubé, Émond et Vandendorpe, 1978 : 35) d'une grande beauté, que même un non-croyant peut apprécier. Dans L'enfant chargé de songes, Anne Hébert s'est inspirée de la Genèse, en présentant l'univers de l'enfance comme un jardin d'Éden : «On aurait pu croire que c'était ça, la vraie vie, une enfance interminable, une sorte de jardin suspendu, entre ciel et terre, où s'ébattaient mère et enfants, à l'abri du mal et de la mort. » (ECS : 37) Cet univers paradisiaque se voit menacé par les «infidélités » des uns et des autres qui viennent troubler l'ordre établi. Mais y a-t-il seulement un ordre possible dans l'univers hébertien ${ }^{13}$ ? La rencontre avec Lydie est à l'image de celle entre Ève et le serpent tentateur. Lydie apparaît sous des traits tantôt séduisants tantôt maléfiques. Dans les rêves de Julien, elle incarne Ève tenant le rôle de la première femme, du premier amour : «Encore un peu elle va franchir la mémoire de Julien, comme Ève franchissant les côtes d'Adam, s'échapper au grand jour ». (ECS : 18) Mais aux yeux de Pauline, Lydie incarne le mal (ECS : 58). La jeune femme se considère d'ailleurs comme le «mauvais génie » (ECS : 59) de Julien et d'Hélène. En pensée, Pauline implore son fils de se souvenir du lieu paradisiaque que représente l'enfance. Elle s'imagine ainsi incarner la femme première : « Je t'ai aimé la première, je suis la première femme de ta vie, comme Ève sous son arbre du bien et du mal, en plein paradis perdu, souviens-toi, c'était l'enfance. » (ECS : 100)

Un autre intertexte avec la Genèse $(G n 4,9)$ semble révélateur de la concurrence que Lydie prend plaisir à installer entre Hélène et Julien. Avant I'arrivée de la jeune femme, frère et sœur passaient tout leur temps ensemble. Lydie sépare les enfants en leur faisant parvenir des lettres qui les poussent à s'isoler pour les lire. Ils souhaitent tous deux être la seule personne à recevoir son affection et son attention. Ainsi, lorsque Pauline demande à sa fille où se trouve son frère et que celle-ci lui répond : «Suis-je la gardienne de mon frère? » (ECS : 69), elle reproduit la réponse à l'Éternel de Caïn, qui, par jalousie, a tué son frère Abel afin d'obtenir toute l'attention et la gratitude de Dieu. Hélène serait-elle prête à sacrifier son frère pour l'amour de Lydie? Elle profitera de la maladie de celui-ci pour se rapprocher de la jeune fille, savourant chaque instant, au grand désarroi de Pauline.

13. L'auteure elle-même a répondu : « Il suffit d'une rencontre pour que tout soit bouleversé. Le monde n'est jamais en ordre. » (Smith, $1983: 56$ ) 
Grandes révélations poétiques

D'abord poète, Anne Hébert a conservé ce « langage plus primitif, qui se compose d'images, et qui va plus vite que la conscience » (Montalbetti, 1969: 39), qu'elle associe à l'écriture des romans poétiques. Il n'est donc pas étonnant d'apprendre que de grands poètes comme Rimbaud, Claudel et Baudelaire figurent parmi ses lectures marquantes. En 1992, elle les intègre dans son roman parfois avec subtilité, parfois un peu plus explicitement. Diverses allusions sont faites à I'œuvre de Rimbaud. Hébert dit d'ailleurs de ce dernier qu'il « est le plus grand poète qu['elle] ai[t] connu. Absolument génial. » (Vanasse, $1982: 443$ ) L'unique poème de Julien est : «Nous entrerons dans des villes splendides / Flambant nus / Montés sur des coursiers d'épouvante. » (ECS: 67) Lorsqu'il le reprend plusieurs années plus tard, le protagoniste modifie le troisième vers : les « coursiers d'épouvante » deviennent « des chevaux d'épouvante » (ECS:127). On peut dire ainsi que son travail renvoie tant à Rimbaud qu'à la Bible. Le poème «Adieu » d'Une saison en enfer se déroule à l'automne. Il est intéressant de noter qu'il s'agit de la saison préférée de Lydie, cellelà même qui fait découvrir à Julien les poètes maudits. Donc, dans le poème rimbaldien, il est écrit : «Et à l'aurore, armés d'une ardente patience, nous entrerons aux splendides villes » (Rimbaud, $1998: 85$ ), ce qui n'est pas sans rappeler le poème de Julien. Pierre Brunel conçoit les «splendides villes » comme «[d]es villes nouvelles qui ne seront pas des hallucinations. » (dans Rimbaud, $1998: 85$ ) Julien aurait aimé que se concrétisent ses fantasmes, et que Lydie lui permette de l'aimer librement. Quant aux « chevaux d'épouvante », ils réfèrent sans doute au Livre du prophète Zacharie (Za 12,4) où Yahweh, le Dieu d'Israël, annonce : «En ce jour-là, - oracle de Yahweh, je frapperai de terreur tous les chevaux, et de délire leurs cavaliers; j'aurai les yeux ouverts sur la maison de Juda, mais je frapperai d'aveuglement tous les chevaux des peuples. » (La sainte Bible, 193914 : 1472) En ce dernier jour, l'Éternel mènera un combat contre les représentants de l'Antéchrist qui auraient trouvé refuge à Jérusalem.

On trouve d'autres citations et allusions se rapportant à l'œuvre de Rimbaud, notamment lorsque Julien et Lydie passent la nuit à la cabane aux renards :

14. Cette édition se trouve dans la bibliothèque personnelle d'Anne Hébert. 
Ils récitent des poèmes à mi-voix. Il parle de son « état primitif de fils du Soleil $\gg{ }^{15}$. Elle dit que c'est pareil pour elle, bien qu'elle soit une fille. Julien jure que c'est à la vie, à la mort. Lydie lui tire les cheveux à pleines poignées, assure qu'elle est vieille comme la terre et la mer ensemble et que ça ne sert à rien de penser au lendemain. (ECS : 98)

La portion de texte en italique est tirée de « Vagabonds » des Illuminations. Le sujet du poème rimbaldien se joue de ce « pauvre frère » (Rimbaud, 1998 : 116) qui entretient toutes sortes de chimères : «Je m'étais joué de son infirmité » (Rimbaud, 1998 : 115-116), en d'autres mots, de sa faiblesse. Il cherche maintenant à se débarrasser de cet homme qui ne cesse de faire le même « songe de chagrin idiot » en le rendant « à son état primitif de fils du soleil » (Rimbaud, $1998: 116$ ). Ce poème vient de nouveau appuyer le caractère diabolique de Lydie, qui ne peut que mener le jeune homme, déjà chargé de rêves, à sa perte en continuant à le nourrir d'illusions. Lydie en a assez de ce petit jeu et choisit d'y mettre un terme, une nuit dans la cabane aux renards, qui est la première et la dernière qu'ils partageront. Après la mort d'Hélène, Julien sera plongé dans un état semi-conscient à l'intérieur duquel le passage du temps n'aura plus d'emprise. Lorsqu'il fréquente Aline, plusieurs années plus tard, celle-ci ne lui procure pas le même état de vertige. Au contraire, à force d'attentions et de tendresse, elle l'ennuie. Le commentaire que lui inspire la lettre d'Aline au ton très maternel va d'ailleurs en ce sens : "Quel ennui I'heure du cher cœur et du cher corps. » (ECS : 145) Cette phrase est la citation presque textuelle du dernier vers du poème «Enfance I » : «Quel ennui, I'heure du "cher corps" et "cher cœur". ${ }^{16} \gg$ (Rimbaud, 1998 : 92) Cet univers rangé qu'Aline souhaite partager avec Julien n'est pas à la hauteur des attentes et des désirs qu'il refoule depuis le départ précipité de Lydie.

Julien semble incapable d'éprouver un bonheur sincère. Assis sur un banc du jardin du Luxembourg, il croit que « [I]a vrai vie n'est pas ici. » (ECS : 143) Il se projette de nouveau dans le passé, comparant ce qu'il voit avec le monde de son enfance. Cette façon de rejeter la réalité et de se convaincre que la vie ne peut se limiter à ses déboires et ses désillusions est partagée par plusieurs personnages hébertiens (de François de la pièce Le temps sauvage à Jean-Éphrem de la Tour d'Un habit de lumière). Tous recherchent ce que pourrait être la vraie vie, celle qui ferait sens

15. Pierre Brunel note que, selon diverses mythologies, cette appellation de « fils du soleil » se rapporte aux immortels (dans Rimbaud, 1998 : 116).

16. Dans une étude du poème rimbaldien, Marcel A. Ruff signale une analogie entre ce vers et deux vers du poème «Le balcon » de Baudelaire : «Car à quoi bon chercher tes beautés langoureuses / Ailleurs qu'en ton cher corps et qu'en ton cœur si doux? » (Rimbaud, $1998: 5$ ) 
et qu'ils ne chercheraient pas à fuir. Cette réflexion sur la vie pourrait renvoyer au poème «Délires I. - Vierge folle. L'Époux infernal » d'Une saison en enfer dans lequel Rimbaud a écrit : «Quelle vie! La vraie vie est absente. Nous ne sommes pas au monde. » (Rimbaud, 1998 : 64) Ainsi, la vierge folle suit l'Époux infernal en enfer, dans cet endroit où la vie est effectivement absente. Julien n'a pas l'impression d'être maître de sa vie. Il pensait que son voyage en France lui permettrait de reprendre le contrôle, mais cela n'est vraisemblablement pas le cas.

Au début des années 1930, Saint-Denys Garneau prête à sa cousine les Cinq grandes odes de Claudel auxquelles elle fait ici référence. On retrouve une allusion au poète dans la première partie du roman. Julien, dans la cathédrale Notre-Dame, « implore la grâce et la révélation » (ECS : 18). Il souhaite être frappé d'une illumination semblable à celle qui a touché Paul Claudel, le 25 décembre 1886, à l'endroit où il se trouvait, et qui a mené le poète français à se convertir au catholicisme. Sa révélation ne viendra que plus tard, après l'annonce de la grossesse d'Aline : «Entre les hommes et Julien il y a ceci de changé qu'il va être le père de l'un d'eux. » (ECS : 153) Cet intertexte, qui prend la forme d'un plagiat selon la classification de Genette, trouve sa source dans la troisième ode, aussi appelée «Magnificat » : «Maintenant entre moi et les hommes il y a ceci de changé que je suis père de l'un d'entre eux. » (Claudel, 1966 : 67) C'est dans cette ode que le sujet célèbre la venue au monde de sa fille Marie.

Anne Hébert cite également quelques titres des Petits poèmes en prose de Baudelaire. Le premier est signalé par l'italique, « Anywhere out of this world », et a subi une légère transformation ${ }^{17}$. Julien ne cherche pas à aller " $n^{\prime}$ importe où hors du monde », il veut simplement quitter son monde ${ }^{18}$. Il cherche à s'éloigner des fantômes qui ne le laissent pas en paix. Ainsi, il se rend en France afin de marcher dans les « pas de Baudelaire ». Dans un même paragraphe, Hébert insère trois titres - le

17. Le titre original étant « Anywhere out of the world».

18. Il souhaite s'évader dans ces lieux entrevus dans les livres: « Anywhere out of this world. Les coupoles de Saint-Pétersbourg, Raskolnikov et Stravoguine [sic], le smog des rues de Londres et les prisons pour dettes de Dickens, Esmeralda dansant sur le parvis de Notre-Dame, le bleu de Chartres (entrevu dans des albums payés très cher), autant d'images qui apparaissent aux yeux de Julien comme les éléments épars de la terre promise dont il rêve. » (ECS : 132) Raskolnikov et Stavroguine sont des personnages des romans de l'écrivain russe Dostoïevski, auteur qu'Anne Hébert mentionne plus d'une fois dans ses entrevues. Le premier personnage prend vie dans Crime et châtiment (1866) et le second, dans Les démons ou Les possédés (1871-1872). Le père de l'auteur anglais Charles Dickens fait de la prison pour dettes en 1824, ce qui marque profondément le fils, qui n'a alors que 12 ans. Ce dernier s'inspire, en 1849-1850, de son père pour créer le personnage de M. Micawber dans David Copperfield. Quant à Esmeralda, il s'agit de I'un des personnages principaux de Notre-Dame de Paris (1831) de Victor Hugo. 
« spleen de Paris », le « mauvais vitrier » et «Mlle Bistouri » à la recherche d'un médecin (ECS : 132) - qui transportent un lecteur averti dans le recueil du célèbre poète. Julien vit le spleen, tente de le contrer, de faire partie du monde à nouveau, mais il est incapable de se dérober à sa langueur et à sa rêverie.

Comme l'a montré Giselle Huot (2007 : 77-100), certains des poèmes d'Anne Hébert présentent des similitudes avec ceux de Saint-Denys Garneau. Ici, Hébert met dans la bouche de Lydie le dernier vers de «Cage d'oiseau » : «Il aura mon âme au bec » (ECS : 88). Elle refuse d'ouvrir son cœur à Julien, de se mettre dans un état de vulnérabilité pour quelqu'un. Elle chiffonne la lettre qu'il lui a écrite «comme quelqu'un qui étouffe un oiseau » (ECS : 88). Elle a peur de perdre le contrôle si elle laisse libre cours à ses sentiments envers lui. Elle préfère le repousser plutôt que de risquer d'être blessée. La plupart des références intertextuelles font ressortir le côté maléfique de la jeune femme. L'auteure montre ainsi la vulnérabilité de Lydie du point de vue psychologique.

Anne Hébert convoque encore un autre poète en reproduisant le premier vers du poème «El Desdichado ${ }^{19}$ » de Gérard de Nerval : «Bientôt [Lydie] se retourne vers [Julien], grave et rêveuse, s'étonne de le trouver si lointain, prisonnier de lui-même, l'appelle doucement, tout bas, pour lui seul, le nomme le Ténébreux, le Veuf, I'Inconsolé. » (ECS : 84) Cette référence donne à penser que Julien est profondément mélancolique de ne pouvoir être avec la femme qu'il désire.

Ainsi, L'enfant chargé de songes rassemble plusieurs lectures marquantes d'Anne Hébert et constitue en ce sens un legs précieux. Dans ce roman, ce sont les personnages qui sont chargés de la quasi-totalité des intertextes et intratextes : Julien en assume plus de la moitié, principalement ceux qui ont trait à la poésie, Lydie et son double, Camille, se partageant la plupart des autres références.

J'ai voulu montrer que le titre du roman relève d'une lecture de jeunesse, L'enfant chargé de chaînes. J'ai ensuite expliqué qu'en remplaçant les chaînes par des songes, l'auteure avait mis l'accent sur les fantasmes intérieurs du personnage principal. Quant aux autres titres envisagés par Anne Hébert, ils marquent une forte intratextualité avec le recueil de poèmes Le jour n'a d'égal que la nuit qu'elle fait paraître la même année. Le relevé des intertextes dans le livre permet de rendre compte de l'importance des lectures d'Anne Hébert dans son processus créateur. Avec L'enfant

19. En français, on pourrait traduire ce titre par « Le malheureux ». 
chargé de songes, l'auteure revient sur sa propre jeunesse sous forme d'allusions aux textes qu'elle a lus avant l'âge de 20 ans et qui n'ont cessé de nourrir son imaginaire. Par l'intertextualité, elle peut travailler sur différents niveaux de sens, symbolique, mythique et allégorique. Les personnages et lieux de son roman s'en trouvent plus riches et complexes; ainsi, I'aspect diabolique de Lydie est-il appuyé par des allusions à Gœthe et à Rimbaud, mais sa vulnérabilité l'est également par des références à Faulkner et à Garneau. Par ailleurs, les nombreux intratextes et intertextes poétiques, notamment Rimbaud, renforcent l'idée qu'Anne Hébert est poète avant tout.

À la différence de la plupart de ses romans et nouvelles, Anne Hébert revisite ici par le songe les paysages de son enfance et redécouvre, par la médiation de son personnage masculin, ceux qui l'ont inspirée un peu plus tard, issus pour la plupart de ses lectures européennes. Dans une entrevue, elle revenait sur son arrivée à Paris en 1954 : « Je m'en souviens très bien. Comme Julien, j'ai vécu cet écran entre mes lectures et la réalité, ce n'était pas tout à fait aussi flambant... beau que ce que j'avais rêvé. Mais petit à petit ça s'est dissipé et j'ai rencontré la réalité, comme Julien. » (Côté, 1992 : E1) Mais Julien y est-il vraiment parvenu? À la différence d'Anne Hébert, celui-ci n'arrivera pas à faire la part des choses et restera prisonnier de ses lectures, de ses songes, et ce, malgré sa volonté de se défaire des images obsédantes de sa mère et de Lydie. 


\section{Bibliographie}

[S.n.] (1939) [1923], La sainte Bible, trad. Augustin Crampon, Paris, Société de Saint Jean l'Évangéliste. BAUDELAIRE, Charles (1968), CEuvres complètes, préface, présentation et notes de Marcel A. Ruff, Paris, Seuil, coll. " L'intégrale ».

CLAUDEL, Paul (1966), Cinq grandes odes suivies d'un Processionnal pour saluer le siècle nouveau; La Cantate à trois voix, préface de Jean Grosjean, Paris, Gallimard, coll. « Poésie ».

COLETTE (1989), « L'étoile Vesper », dans Romans - Récits - Souvenirs (1941-1949). Critique dramatique (1934-1938), Paris, Robert Laffont, coll. « Bouquins », tome III : 581-684.

CÔTÉ, Lucie (1992), « Anne Hébert. La vie d'abord », La Presse, 28 mai : E1.

DUBÉ, Cécile, Maurice ÉMOND et Christian VANDERDOPE (1978), « Anne Hébert : entrevue », Québec français, $\mathrm{n}^{\circ}$ 32, décembre : 33-35.

ESCOMEL, Gloria (1980), « Anne Hébert : 30 ans d'écriture », Madame au foyer, septembre : 6-22.

FORTIN, Marie-Claude (1992), « Anne Hébert : une saison en enfance », Voir, Montréal, vol. 6, n²7, 4 au 10 juin : 22.

GENETTE, Gérard (1982), Palimpsestes : la littérature au second degré, Paris, Seuil, coll. « Poétique ». HÉBERT, Anne (1958), Les chambres de bois, Paris, Seuil.

HÉBERT, Anne (1967), Le temps sauvage, La mercière assassinée, Les invités au procès, Montréal, $\mathrm{HMH}$.

HÉBERT, Anne (1970), Kamouraska, Paris, Seuil.

HÉBERT, Anne (1972) [1963], Le torrent, nouvelle édition suivie de deux nouvelles inédites, Montréal, $\mathrm{HMH}$, coll. « L'arbre ».

HÉBERT, Anne (1975), Les enfants du Sabbat, Paris, Seuil.

HÉBERT, Anne (1976), « Mon cœur sauvage, je le dis en français », Les nouvelles littéraires, $\mathrm{n}^{\circ} 2517$, 29 janvier : 22.

HÉBERT, Anne (1982), Les fous de Bassan, Paris, Seuil.

HÉBERT, Anne (1999) [1992], L'enfant chargé de songes, Paris, Seuil, coll. « Points », n 615.

HÉBERT, Anne (1999), Un habit de lumière, Paris, Seuil.

HÉBERT, Anne (2013), OEuvres complètes I. Poésie, édition établie par Nathalie Watteyne, suivi de Dialogue sur la traduction à propos du Tombeau des rois, édition établie par Patricia Godbout, Montréal, Presses de l'Université de Montréal, coll. « Bibliothèque du Nouveau Monde ».

HUOT, Giselle (2007), « De quelques similitudes, différences et influences : de Saint-Denys Garneau Anne Hébert », Les Cahiers Anne Hébert, $n^{\circ} 7: 77-100$.

JACQUES, Paula et Richard HULOT (1995), [Entrevue avec Anne Hébert], Pentimento, Paris, France Inter, émission de radio (65 min.), 15 janvier.

KAUFFMANN, Jean-Paul (1970), « De Paris, elle réinvente le Québec », La Presse (cahier Perspectives), 12 décembre : 8; 10. 
LAMONTAGNE, Marie-Andrée (1993), « Voyage avec Saint-Denys Garneau », Liberté, vol. 35, $\mathrm{n}^{\circ}$ 4-5 (208-209) : 210-215.

MALLARMÉ, Stéphane (1993) [1976], Igitur; Divagations; Un coup de dés, préface Yves Bonnefoy, Paris, Gallimard, coll. « Poésie », n 113.

MAURIAC, François (1978), "L'enfant chargé de chaînes ", dans CEuvres romanesques et théâtrales complètes, préparé par Jacques Petit, Paris, Gallimard, coll. « Bibliothèque de la Pléiade », tome 1 : 1-79.

MITCHELL, Constantina T. et Paul R. CÔTÉ (1996), « Voices, Dreams and Narrative Organization: Anne Hébert's L'enfant chargé de songes », dans Constantina T. Mitchell et Paul R. Côté (dir.), Shaping the Novel: Textual Interplay in the Fiction of Malraux, Hébert, and Modiano, Providence, Berghahn Books : 95-158.

MONTALBETTI, Jean (1969), « Anne Hébert. Un poète comme tout le monde », Le magazine littéraire, $\mathrm{n}^{\circ} 35$, décembre : 38-39.

NIJBOER, Catrien (1997), "L'interartistique dans l'œuvre d'Anne Hébert : L'enfant chargé de songes », dans Madeleine Ducrocq-Poirier [et al.], Anne Hébert, parcours d'une œuvre. Actes du colloque de la Sorbonne, Montréal, l'Hexagone : 397-410.

PERREAULT, Luc (1966), « Anne Hébert : “On s'est fait de moi une image arrêtée” », La Presse, 24 septembre : $7 ; 12$.

RIMBAUD, Arthur (1998), Une saison en enfer. Illuminations et autres textes (1873-1875), édition de Pierre Brunel, Paris, Le livre de poche, coll. « Classiques de poche », n 9636.

ROYER, Jean (1980), « Anne Hébert : jouer avec le feu », Le Devoir, 26 avril : 21-22.

S[ALGAS], J[ean]-P[ierre] (1985), « II faut sortir du ghetto », La quinzaine littéraire, spécial « Écrire les langues françaises $», n^{\circ} 436,16-31$ mars : 18.

SMITH, Donald (1983), "Anne Hébert et les racines de l'imaginaire », L'écrivain devant son œuvre : entrevues, Montréal, Québec Amérique, coll. «Littérature d'Amérique » : 35-58.

STIERLE, Karlheinz (1977), "Identité du discours et transgression lyrique », Poétique, $n^{\circ} 32$, novembre : 422-441.

TADIÉ, Jean-Yves (2005) [1994], Le récit poétique, Paris, Gallimard, coll. « Tel ».

VANASSE, André (1982), " L'écriture et l'ambivalence, entrevue avec Anne Hébert », Voix et images, vol. VII, $n^{\circ} 3$, printemps : 441-448.

WATTEYNE, Nathalie (2008), « Les lectures d’Anne Hébert », Les écrits, n 123, août : 53-73. 